\title{
APPLICATION OF THE RECIPROCAL THEOREM TO SOME PROBLEMS FOR THE ELASTIC HALF-SPACE
}

\author{
J. R. BARBER \\ Department of Mechanical Engineering and Applied Mechanics, University of Michigan, \\ Ann Arbor, MI 48109, U.S.A. \\ and \\ F. A. Sturla \\ Analysis Methods, General Motors Technical Center, 30200 Mound Road, Warren, MI 48090. U.S.A. \\ (Received 26 July 1990 ; in retised form 20 November 1990 )

\begin{abstract}
BETTI's reciprocal theorem is used to determine the normal displacements duc to a normal point force at the plane surface of a generally-anisotropic half-space or a transversely-isotropic general elastic foundation. using a state of plane sinusoidal loading as auxiliary solution. The method is also extended to give a dual integral equation formulation of the axisymmetric contact problem for an inhomogeneous half-space in which the elastic moduli are arbitrary functions of depth.
\end{abstract}

\section{INTRODUCTION}

BETrI's reciprocal theorem can often be used to obtain specific results to problems in elasticity without obtaining a complete solution for the stress and displacement fields. Indeed, in many cases results can be obtained for problems in which a complete solution would be impossible. Since the theorem leads to an integral relation, the most common applications are those in which force resultants, average displacements or volume changes are required. For example, Mossakowskir (1953) and SHIELD (1967) show how it can be used to express the force and moment resultants on a rigid punch of arbitrary profile in terms of integrals involving the profile function and the pressure distribution under a flat punch of the same plan-form.

The results generated by the reciprocal theorem depend on the choice of auxiliary solution, which must in general represent a sufficiently simple state of the system for the stress and displacement fields to be obtainable in closed form. In the present paper, we shall explore the consequences of using as auxiliary solution a state in which the fields vary sinusoidally with one spatial coordinate. As might be anticipated, this leads to results bearing some similarity to those of Fourier transformation, but, in general, the use of the reciprocal theorem provides a much more efficient formulation.

We shall apply the method to two special cases - the homogeneous generallyanisotropic half-space and the general elastic foundation (GLADWELL, 1980, §11.1), 
which includes the case of a transversely-isotropic half-space or layer in which the elastic properties are permitted to vary arbitrarily with depth.

\section{The Auxillary Solution}

The half-space or foundation will be taken to occupy the region $z>0$ in Cartesian coordinates $(x, y, z)$, the loaded surface of interest being the plane $z=0$. For the auxiliary solution, we consider the case in which this surface is loaded by a sinusoidal normal traction

$$
\begin{aligned}
& \sigma_{z z}(x, y, 0)=S \cos (a x), \\
& \sigma_{z x}(x, y, 0)=\sigma_{z y}(x, y, 0)=0
\end{aligned}
$$

for all $(x, y)$.

If the elastic properties of the half-space are independent of $x$ and $y$, the resulting stress and displacement components will have the generalized form

$$
\sigma, u(x, y, z)=f_{1}(z) \cos (a x)+f_{2}(z) \sin (a x),
$$

where $f_{1}$ and $f_{2}$ are functions of $z$ to be determined from the equilibrium and elasticity equations for the material and from the boundary conditions (1) and (2). Since the functions to be determined depend on only one variable, this problem will reduce to a set of ordinary linear differential equations $\dagger$ which can be solved by various methods. For our purpose, we only need to know the normal surface displacement due to the traction distribution (1) and (2), which we can express in the form

$$
u_{z}(x, y, 0)=S g(a) \cos (a x)
$$

where we have recognized the linearity of the system by extracting the loading parameter $S$ explicitly.

The omission of a sin (ax) term in (4) might seem to imply the assumption of a symmetry unjustified in the case of general anisotropy. However, if we assume the more general expression $S\left[g_{\mathrm{I}}(a) \cos (a x+b)\right]$ for the displacement $u_{z}(x, y, 0)$ and then apply the reciprocal theorem to this solution and to the same solution with $x$ replaced by $x+c$, we obtain an equation which can only be satisfied for all $c$ if $b=n \pi$, where $n$ is an integer, thus proving that the normal surface displacement and traction must be in phase for the most general linear elastic foundation.

\section{The General Anisotropic Half-SPace}

In this section, we consider the case where the half-space is homogeneous, but generally-anisotropic-i.e. the 21 independent elastic constants may take arbitrary values. 


\subsection{Willis' results}

Some remarkably simple results for surface loading of such a half-space have been developed by WiLLIS (1966). He first notes that the fundamental problem of the loading of the half-space by a concentrated normal force, $F$, is self-similar. It follows from equilibrium considerations that the stress field must vary with distance $R$ from the point of application of the force with $R^{-2}$, which implies that the displacement field varies with $R^{-1}$. In particular, we conclude that, if the force is applied at the origin, the normal surface displacement must take the form

$$
u_{z}(x, y, 0)=\frac{F h(\theta)}{r},
$$

where $r=\sqrt{x^{2}+y^{2}}, \tan (\theta)=y / x$, and $h(\theta)$ is an unknown function to be determined, which will of course be a constant in the special case of transverse isotropy.

We can use this equation as a Green's function to determine the displacement due to the distributed contact traction

$$
\sigma_{z z}(x, y, 0)=-\frac{p_{0}}{\sqrt{1-\left(\frac{x}{a}\right)^{2}-\left(\frac{y}{b}\right)^{2}}}
$$

within the elliptical region of semi-axes $a$ and $b$. A convenient formulation is to define polar coordinates on the field point as origin as shown in Fig. 1, in which case we obtain

$$
u_{z}(P)=\int_{0}^{\pi} \int_{A(\theta)}^{B(\theta)} \frac{p_{0} h(\theta) \mathrm{d} s \mathrm{~d} \theta}{\sqrt{1-\left(\frac{x}{a}\right)^{2}-\left(\frac{y}{b}\right)^{2}}},
$$

where $P$ is the point $\left(x^{\prime}, y^{\prime}\right)$, and $x=x^{\prime}+s \cos \theta, y=y^{\prime}+s \sin \theta$.

The inner integral is the reciprocal of the square root of a quadratic function of $s$ between its two singular points, and hence can be written in the standard form

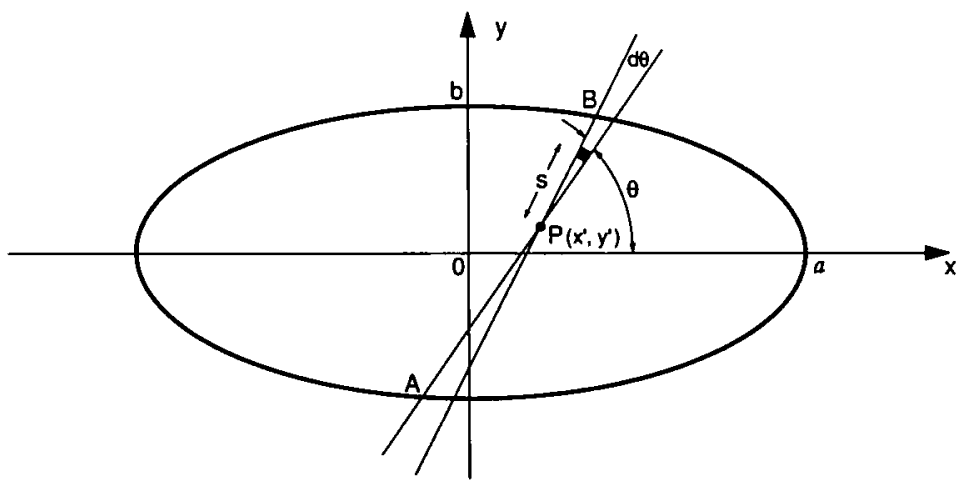

FIG. 1. Integration of (7) using polar coordinates based on the field point $P$ as origin. 


$$
C(\theta) \int_{A(\theta)}^{B(0)} \frac{\mathrm{d} s}{\sqrt{1-\left(\frac{x}{a}\right)^{2}-\left(\frac{y}{b}\right)^{2}}}=\int_{-c}^{c} \frac{\mathrm{d} z}{\sqrt{c^{2}-z^{2}}}=\pi,
$$

using a simple linear change of variable, where $C(\theta)=\left[(\cos (\theta) / a)^{2}+(\sin (\theta) / b)^{2}\right]$. Equation (7) therefore reduces to

$$
u_{z}(P)=\pi p_{0} \int_{0}^{\pi} \frac{h(\theta) \mathrm{d} \theta}{C(\theta)},
$$

which is clearly independent of $x^{\prime}, y^{\prime}$, showing that (6) describes the traction distribution under a flat rigid punch of elliptical plan-form, indenting the generallyanisotropic half-space.

A similar integration procedure can be used to demonstrate that the more general traction distribution

$$
\sigma_{z z}(x, y, 0)=\frac{P_{n}(x, y)}{\sqrt{1-\left(\frac{x}{a}\right)^{2}-\left(\frac{y}{b}\right)^{2}}}
$$

within the elliptical region produces the displacement profile

$$
u_{z}(P)=Q_{n}\left(x^{\prime}, y^{\prime}\right)
$$

within the elliptical region, where $P_{n}$ and $Q_{n}$ are polynomials of degree $n$ in $x, y$, and $x^{\prime}, y^{\prime}$, respectively. This is known as Galin's theorem (WILLIS, 1967) - see also GLADWELL $(1980, \$ 12.2)$ for additional references and an alternative method of proof). The special case $n=2$ corresponds to the Hertzian contact of smooth quadratic surfaces. Willis used this result to deduce that the Hertzian contact area would remain elliptical and the traction distribution be given by the classical expression

$$
\sigma_{z z}(x, y, 0)=-p_{0} \sqrt{1-\left(\frac{x}{a}\right)^{2}-\left(\frac{y}{b}\right)^{2}}
$$

for the case of general anisotropy, though the inclination and magnitude of the semiaxes of the ellipse would of course be affected by the function $h(\theta)$.

\subsection{Determination of the function $\mathrm{h}(\theta)$}

To obtain more specific results, we need to evaluate the function $h(\theta)$. WiLLIs (1966) solved this problem by performing a double Fourier transformation on the delta function loading, and hence his results for the surface displacements require the numerical inversion of a corresponding contour integral. We shall show in this section that the reciprocal theorem can be used to relate $h(\theta)$ directly to the solution of the simpler auxiliary problem of Section 2.

We establish a preliminary result by considering: (i) a normal force $F$ applied at the origin, and (ii) an equal force applied at the point $P(r, \theta)$. Noting that the halfspace is homogeneous, and using (5), we conclude that the normal displacement at $P$ 
due to (i) is $F h(\theta) / r$ and that at $O$ due to (ii) is $F h(\theta+\pi) / r$. These must be equal by Maxwell's reciprocal theorem. It follows that $h(\theta)=h(\theta+\pi)$ and hence that the function $h(\theta)$ permits the Fourier expansion

$$
h(\theta)=\sum_{i=0}^{\infty} A_{i} \cos (2 i \theta)+\sum_{i=1}^{\infty} B_{i} \sin (2 i \theta)
$$

We next apply Betti's theorem to : (i) the concentrated force $F$ applied at the origin, and (ii) the sinusoidal solution of Section 2. The theorem states that

$$
\int_{S} \mathbf{t}_{(\mathrm{i})} \cdot \mathbf{u}_{(\mathrm{ii})} \mathrm{d} S=\int_{S} \mathbf{t}_{(\mathrm{ii})} \cdot \mathbf{u}_{(\mathrm{i})} \mathrm{d} S,
$$

where $\mathbf{t}$ and $\mathbf{u}$ are, respectively, the traction and displacement at the surface $S$, which in this instance consists of the plane $z=0$.

Substituting for the tractions and displacements from (1), (2), (4) and (5) and cancelling a factor $F S$, we obtain $\dagger$

$$
\begin{aligned}
g(a)= & \int_{0}^{\infty} \int_{0}^{2 \pi} h(\theta) \cos (\operatorname{arcos} \theta) \mathrm{d} \theta \mathrm{d} r \\
= & \sum_{i=0}^{\infty} A_{i} \int_{0}^{x} \int_{0}^{2 \pi} \cos (\operatorname{arcos} \theta) \cos (2 i \theta) \mathrm{d} \theta \mathrm{d} r \\
& +\sum_{i=1}^{\infty} B_{i} \int_{0}^{\infty} \int_{0}^{2 \pi} \cos (\operatorname{arcos} \theta) \sin (2 i \theta) \mathrm{d} \theta \mathrm{d} r,
\end{aligned}
$$

using (13).

The second term in this expression is zero by symmetry and the first can be evaluated using 3.715.18 and 6.511.1 of GRADSHTEYN and RYZHIK (1980) to obtain

$$
\begin{aligned}
g(a) & =\frac{2 \pi}{a} \sum_{i=0}^{\infty} A_{i} \cos (n \pi) \\
& =\frac{2 \pi}{a} h\left(\frac{\pi}{2}\right)
\end{aligned}
$$

from (13).

Since this result is true for all $a$, it shows that $g(a)$ must vary with $a^{-1}$-a fact which could have been established directly using dimensional and similarity arguments. More importantly, it establishes a simple algebraic relation between the unknown function $h(\theta)$ and the essentially one-dimensional solution of the problem of Section 2.

Furthermore, since the original coordinate system was chosen arbitrarily, (17) can be used to determine the function $h$ for any value of $\theta=\alpha$. All that is necessary is

$\dagger$ Equation (15) is based on the assumption that only the tractions and displacements on the free surface $z=0$ make a contribution to the integrals in (14). This can be verified by considering the finite body bounded additionally by the hemispherical surface $R=b$. In the limit $a b \gg 1$, the contribution from this surface reduces to a series of Bessel functions of the form $J_{n}(a b)$ which all decay to zero with $(a b)^{-1 / 2}$ as $a b \rightarrow 0$. 
to choose the auxiliary solution in such a way that the ridges of the sinusoidal distribution are inclined at $\alpha$ to the $y$-axis. Formally, this implies replacing $\cos (a x)$ in (1) by $\cos [a(x \sin \alpha-y \cos \alpha)]$.

Thus, the problem of determining $h(\theta)$, and hence the Green's function (5) for normal surface loading of the generally-anisotropic half-space, is reduced to the solution of the auxiliary problem of Section 2. Except in certain cases where the characteristic equation has repeated roots, this solution can be written down using functions of the form exp (iaz) in the formalism due to STROH (1958) and EsHELBY et al. (1953). Alternatively, a more direct solution is to write the equilibrium equations in terms of the three displacement components $u_{x}, u_{y}, u_{z}$, each of which can be written in the form (3). After separating sine and cosine terms, this leaves six linear ordinary differential equations with constant coefficients which can be reduced to a single sixthorder equation. The virtue of this method is that the special cases appear naturally as repeated differential factors in the final equation and can be treated by standard methods.

Both solutions involve considerable algebraic complexity and hence no further details are given here. However, the manipulations are essentially routine and can readily be performed using modern symbolic processors.

\section{The Inhomogeneous HalF-SPACE}

We now turn our attention to the problem of the half-space whose elastic properties vary with depth $z$, which includes as special cases the elastic layer supported on a rigid foundation or a composite layer made up of several separately homogeneous layers. In such cases, the layer(s) may be bonded to the substrate or there may be frictionless contact, but we exclude cases in which separation occurs at the interface, since this would lead to additional terms in the expression obtained from the reciprocal theorem.

The problem of the general elastic foundation has received extensive consideration, particularly in the Soviet literature, good surveys of this work being given by Gladwell $(1980, \$ 11.1)$ and Popov (1972). Foundations in which the elastic properties vary continuously with depth are of special interest in soil mechanics. The complete solution of two- and three-dimensional problems of this type is very difficult and attention has been largely restricted to cases where the elastic moduli vary with a power of the depth and dimensionless ratios such as Poisson's ratio are constant. Much of this work is summarized by GLADWELL $(1980, \S 12.5)$.

\subsection{The point force solution}

In this section, no restrictions are imposed on the nature of the variation of elastic properties with depth, except that the half-space will be assumed to be transversely isotropic, so that axisymmetric problems will have axisymmetric solutions. In particular, if the foundation is loaded by a concentrated normal force $F$, the fields will be axisymmetric, but the problem is no longer self-similar since the depth variation of the elastic properties introduces its own length scale. Thus, (5) is replaced by the relation 


$$
u_{z}(x, y, 0)=F \psi(r),
$$

where $\psi(r)$ is an unknown function of $r$ to be determined.

As before, the auxiliary solution will be defined by (1), (2) and (4), with solution (5), but we note that the symmetry of the material now ensures a plane strain solution and also permits us to replace (3) by a form involving a single unknown function with a sine or cosine multiplier, depending on the symmetry of the corresponding component. Thus, the displacement field in the auxiliary solution is defined by two functions $f_{1}$ and $f_{2}$ through the relations

$$
u_{x}=f_{1}(z) \sin (a x), \quad u_{y}=0, \quad u_{z}=f_{2}(z) \cos (a x)
$$

and the two nontrivial equilibrium equations will yield two linear ordinary differential equations for $f_{1}$ and $f_{2}$ with variable coefficients. Alternatively, the stress field can be represented by an Airy stress function of the form $\phi=f(z) \cos (a x)$, in which case the compatibility equation provides a single linear ordinary differential equation with variable coefficients for the unknown function $f(z)$.

Applying the reciprocal theorem (14) to the point force solution defined by (18) and this auxiliary solution and cancelling a factor $F S$, we obtain

$$
\begin{aligned}
g(a) & =\int_{0}^{\infty} \int_{0}^{2 \pi} \psi(r) \cos (a r \cos \theta) r \mathrm{~d} \theta \mathrm{d} r \\
& =2 \pi \int_{0}^{\infty} r \psi(r) J_{0}(a r) \mathrm{d} r,
\end{aligned}
$$

using 3.715.18 of GRADSHTEYN and RYZHIK (1980).

This result can be expressed in the Hankel transform notation of GLADWELL (1980) as

$$
g(a)=2 \pi \mathscr{H}_{0}[\psi(a) ; r]
$$

which has the inversion

$$
\psi(r)=\frac{1}{2 \pi} \mathscr{H}_{0}[g(r) ; a] \equiv \frac{1}{2 \pi} \int_{0}^{\infty} a g(a) J_{0}(a r) \mathrm{d} a
$$

(GLADWELL, 1980, §5.6). Substitution in (18) completes the solution for the normal surface displacement due to the point force.

\subsection{Application to contact problems}

So far we have concentrated on the use of the reciprocal theorem to determine the surface displacement due to a point force. Once obtained, this result can of course be used as a Green's function to formulate contact problems for the half-space or foundation as in Section 3.1. However, in certain cases, the reciprocal theorem can be used directly to formulate the contact problem without first obtaining the Green's function. 
As an example, consider the classical frictionless axisymmetric contact problem defined by the boundary conditions

$$
\begin{gathered}
u(r) \equiv u_{z}(r, 0)=u_{0}(r) ; \quad 0<r<b \\
p(r) \equiv-\sigma_{z z}(r, 0)=0 ; \quad r>b \\
\sigma_{z \theta}=\sigma_{z r}=0 \quad \text { all } r
\end{gathered}
$$

at the surface $z=0$ of the inhomogeneous half-space.

Applying the reciprocal theorem to this problem, using the auxiliary solution of Section 2, we obtain

$$
\int_{0}^{\infty} \int_{0}^{2 \pi} r p(r) g(a) \cos (a r \cos \theta) \mathrm{d} \theta \mathrm{d} r=\int_{0}^{\infty} \int_{0}^{2 \pi} r u(r) \cos (a r \cos \theta) \mathrm{d} \theta \mathrm{d} r
$$

which on performance of the inner integrals reduces to

$$
g(a) \mathscr{H}_{0}[p(a) ; r]=\mathscr{H}_{0}[u(a) ; r] \equiv \phi(a) .
$$

Both $p(r)$ and $u(r)$ can then be expressed in terms of the new function $\phi(a)$ by inverting the transforms in (28) with the result

$$
\begin{aligned}
& u(r)=\mathscr{H}_{0}[\phi(r) ; a], \\
& p(r)=\mathscr{H}_{0}\left[\frac{r \phi(r)}{g(r)} ; a\right] .
\end{aligned}
$$

The boundary conditions (24) and (25) then lead to the pair of dual integral equations

$$
\begin{aligned}
\mathscr{H}_{0}[\phi(r) ; a] & =u_{0}(r) ; \quad 0<r<b, \\
\mathscr{H}_{0}\left[\frac{r \phi(r)}{g(r)} ; a\right] & =0 ; \quad r>b .
\end{aligned}
$$

Defining

$$
\chi(x)=\mathscr{F}_{0}\left[\frac{a \phi(a)}{g(a)} ; x\right] \equiv\left(\frac{2}{\pi}\right)^{1 / 2} \int_{0}^{x} \frac{a \phi(a)}{g(a)} \cos (a x) \mathrm{d} a
$$

as the Fourier cosine transform of $[a \phi(a) / g(a)]$ and using 5.7.17 and 5.7.20 of Gladwell (1980), we deduce that (32) will be satisfied provided

$$
\chi(x)=0 ; x>b
$$

The inversion of (33) then yields

$$
\left(\frac{2}{\pi}\right)^{1 / 2} \int_{0}^{b} \chi(x) \cos (x a) \mathrm{d} x=\frac{a \phi(a)}{g(a)}
$$

which can be substituted into (31) to give 


$$
u_{0}(r)=\left(\frac{2}{\pi}\right)^{1 / 2} \int_{0}^{\infty} \int_{0}^{b} g(a) \chi(x) \cos (x a) J_{0}(a r) \mathrm{d} x \mathrm{~d} a ; \quad 0<x<b .
$$

By changing the order of integration, this can be written as an integral equation of the first kind for $\chi(x)$, i.e.

$$
\int_{0}^{b} \chi(x) K(x, r) \mathrm{d} x=u_{0}(r) ; \quad 0<r<b,
$$

where

$$
K(x, r) \equiv\left(\frac{2}{\pi}\right)^{1 / 2} \int_{0}^{\infty} g(a) \cos (x a) J_{0}(a r) \mathrm{d} a .
$$

Once $\chi(x)$ is determined, the normal stress and displacement components at the surface - in particular the contact pressure - can be recovered from (29), (30) and (34).

\section{Conclusions}

The above results show that Betti's reciprocal theorem can be used to obtain several important relations between the surface tractions and displacements in bodies with fairly general constitutive laws, for which a solution for the complete fields would be very tedious.

\section{ACKNOWLEDGEMENTS}

J. R. Barber is pleased to acknowledge support from the National Science Foundation under Contract number MSM-8820623.

\section{REFERENCES}

Eshelby, J. D., Read, W. T. and SHOCKLEY, W.

Gladwell, G. M. L.

GRADSHTEYN, I. S. and

RYZHIK, I. M.

Mossakowskil, V. I.

Popov, G. IA.

SHIELD, R. T.

STROH, A. N.

WILLIS, J. R.

WILLIS, J. R.
1953 Acta Metall. 1, 25I.

1980 Contact Problems in the Classical Theory of Elasticity. Sijthoff \& Noordhoff, Alphen aan den Rijn.

1980 Tables of Integrals, Series and Products. Academic Press, New York.

$1953 P M M 17,477$ (in Russian).

1972 Soviet appl. Mech. 8, 237.

1967 Z. angew. Math Phys. 18, 682.

1958 Phil. Mag. 3, 625.

1966 J. Mech. Phys. Solids 14, 163.

1967 J. Mech. Phys. Solids 15, 331. 